14

\title{
Разработка ЯМР-релаксометра для определения динамики намагниченности протонов воды живых тканей и его использование для оценки возрастных изменений
}

\author{
(С) Ю.И. Неронов, Д.Д. Косенков
}

Всероссийский научно-исследовательский институт метрологии им. Д.И. Менделеева, 190005 Санкт-Петербург, Россия

Университет ИТМО,

193101 Санкт-Петербург, Россия

e-mail: yineronov@mail.ru

Поступило в Редакцию 5 декабря 2018 г.

В окончательной редакции 24 января 2019 г.

Принято к публикации 29 января 2019 г.

Разработан настольный ЯМР-релаксометр, позволяющий определять времена спин-спиновой релаксации протонов воды живых тканей. Времена спиновой релаксации протонов воды отражают изменения среднестатистического числа парамагнитных центров, поскольку их присутствие увеличивает скорость восстановления намагниченности протонов воды живой ткани в десятки раз. Этот метод, в частности, позволил регистрировать возрастные изменения (саркопению), связанные с замещением мышечного волокна соединительной тканью. Подобные релаксометры комфортны в использовании и являются перспективными для анализа и диагностики изменений и нарушений метаболических процессов.

DOI: 10.21883/JTF.2019.07.47810.424-18

\section{Введение}

Основой существования живого организма является метаболизм, представляющий собой совокупность взаимосвязанных химических реакций с участием десятков тысяч молекул и ионных соединений. Направленность этих реакций обеспечивает обновление клеточных структур живых тканей. Задачей медицинского обслуживания является обнаружение таких начальных патологических отклонений в организме человека, которые могут переходить в существенные нарушения функционирования живых тканей. В этой связи требуется разработка таких широко доступных приборов, которые в совокупности позволят формировать цифровой портрет физиологического состояния тканей живого организма для своевременного выявления необратимых изменений.

Современные ЯМР-томографы со сверхпроводящими магнитами являются дорогостоящими приборами, но позволяют хорошо визуализировать органы живого организма и оценивать патологические отклонения. Контрастное отличие соседних органов в таких приборах обеспечивается, прежде всего, тем, что разные органы имеют клеточные структуры, у которых отличаются времена спиновой релаксации протонов воды. Времена релаксации оценивают постоянными $T_{1}$ и $T_{2}$ (спинрешеточная и спин-спиновая релаксации), которые определяют динамику протонной намагниченности. Эти постоянные зависят от концентрации парамагнитных включений, находящихся во внутриклеточной и внеклеточной воды того или иного органа.

Времена релаксации протонов в чистой воде равны: $T_{1}\left(\mathrm{H}_{2} \mathrm{O}\right)=3.61 \mathrm{~s} ; T_{2}\left(\mathrm{H}_{2} \mathrm{O}\right)=1.97 \mathrm{~s}\left(t=20^{\circ} \mathrm{C}\right)$ и отра- жают скорость релаксации протонов за счет взаимодействия молекул воды друг с другом в отсутствие примесей и, в частности, в отсутствие кислорода, растворенного в воде, обладающего парамагнитными свойствами. Если воздух, содержащий кислород, не удаляется из воды, времена релаксации протонов уменьшаются: $T_{1} \approx 1.68 \mathrm{~s}$ и $T_{2} \approx 1.05 \mathrm{~s}$. Известно, что растворимость кислорода в воде при $20^{\circ} \mathrm{C}$ составляет $\approx 9.1 \mathrm{mg} / \mathrm{l}$. В этом случае на одну молекулу растворенного кислорода $\mathrm{O}_{2}$ приходится $2 \cdot 10^{5}$ молекул $\mathrm{H}_{2} \mathrm{O}$. Из этого примера следует, что времена релаксации протонов воды очень чувствительны к присутствию даже небольшого числа парамагнитных включений.

Из-за влияния всего набора непарных магнитных моментов электронов, присутствующих в сложной среде живой ткани, времена релаксации протонов воды снижаются в десять или более раз. Известное соотношение [1-3] позволяет качественно описывать этот механизм релаксации следующим выражением:

$$
1 / T 1=\left[\left(4 \pi^{2} \gamma^{2} \eta\right) / k T\right]\left\{\Sigma\left[\Sigma\left(\mu_{i}\right)^{2}\right]\right\},
$$

где $\gamma$ - гиромагнитное отношение протона, $\eta$ вязкость среды, $k T$ - постоянная Больцмана и температура, $\Sigma\left(\mu_{i}\right)^{2}$ - сумма средних квадратов по всем $\mu_{i}$ $\left(\mu_{i}\right.$ - эффективные магнитные моменты молекулярных структур или ионов, имеющих неспаренные электроны). В живой ткани носителями эффективных магнитных моментов неспаренных электронов являются кислород и ряд его активных форм; различные свободные радикалы; некоторые ферменты биохимических реакций, ионы и ионные соединения с атомами металлов, такими как 
ионы железа, меди и т.д. Второе суммирование в уравнении (1) описывает необходимость учета ограниченного времени жизни $i$-го метаболита, имеющего $\mu_{i}$.

Время жизни некоторых активных метаболитов может быть очень малым, и их влияние на спиновую релаксацию протонов воды соответственно невелико. Однако их количество в исследуемой живой ткани может быть достаточно большим, чтобы существенно влиять на скорость восстановления равновесного состояния протонной намагниченности.

\section{Описание экспериментальной установки}

Ранее в работах [4-7] сообщалось о разработке миниЯМР-томографа с использованием постоянного настольного магнита с полем $B=0.128 \mathrm{~T}$. Применение такого типа прибора оказывается более эффективным в режиме регистрации времени релаксации [8]. Причем если в подобных ЯМР-релаксометрах обеспечить линейность регистрации амплитуды сигналов (на уровне $d A / A<1 \%$ ), то параметры $T_{1}$ и $T_{2}$ могут регистрировать динамику метаболических процессов.

В настоящем варианте нашего прибора ЯМР-сигналы спинового эха регистрировались с помощью резонансной индуктивности поверхностного типа. Она изготовлена в виде кольцевой намотки, содержащей 20 проводников (диаметр кольца $\approx 27 \mathrm{~mm}$ ) и имеющей изгиб для оптимального полуохвата цилиндрической ампулы или участка мышечной ткани ладони участника эксперимента (рис. 1). Резонансная индуктивность была экранирована от исследуемого образца электростатическим экраном. Экран представляет собой изогнутую плоскость, содержащую набор тонких проводников, заземленных с одного конца. Такой экран не уменьшает

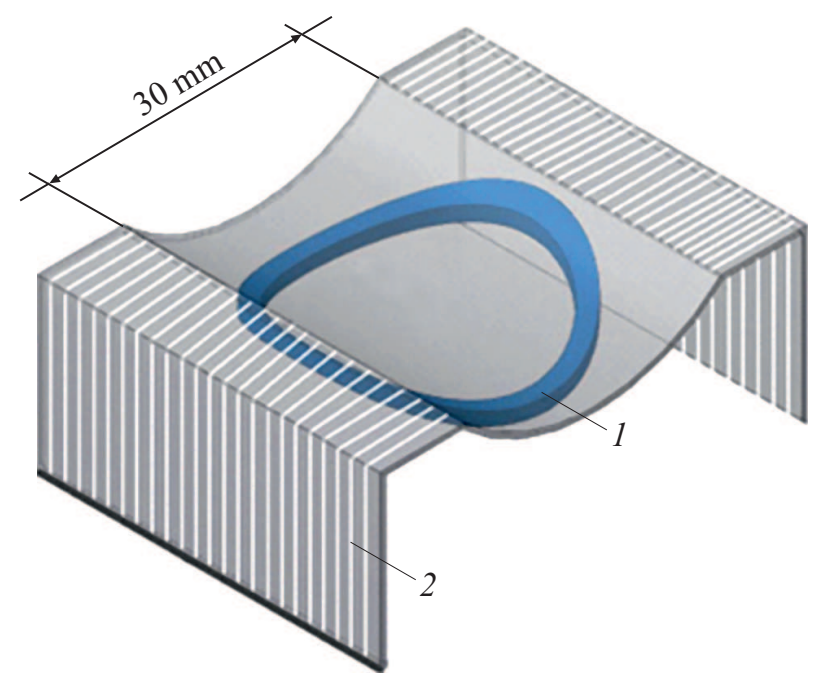

Рис. 1. Форма катушки датчика ЯМР-сигналов: 1 - расположение витков резонансной индуктивности; 2 - электростатический экран. добротность резонансного контура и позволяет сохранять стабильные условия с оптимальными настройками радиочастотного резонанса при исследовании объектов, имеющих разнообразную форму.

Электронная часть прибора [8] была заменена на модуль, собранный на современных интегральных микросхемах. Для формирования частот резонанса была использована микросхема AD9958, которая синтезирует опорные частоты, обеспечивающие регистрацию ЯМРсигналов от ядер двух типов - протонов и дейтронов (как в работах $[9,10])$. Сигналы от дейтронов формировались от дополнительного образца, расположенного в магните, и использовались для автоматической подстройки минимизации температурного дрейфа магнитного поля и повышения фазовой стабильности ЯМР эхосигналов на протонном резонансе. Прибор потребляет энергию не более $30 \mathrm{~W}$, имеет компактное исполнение и может управляться от небольшого ноутбука.

\section{Методика определения времени релаксации и оценка погрешности}

Для оценки диагностических возможностей ЯМРрелаксометра в исследовании живых тканей было разработано программное обеспечение, которое обеспечивало формирование требуемых последовательностей импульсов и регистрацию ЯМР эхо-сигнала. Программное обеспечение содержало арифметический блок для расчета как времени релаксации, так и погрешности результата. Мы ограничивались определением констант $T_{2}$, поскольку они более удобны для регистрации, чем $T_{1}$. Причем константы $T_{2}$ не менее чувствительны к динамике биохимического состава живых тканей, чем $T_{1}$.

На рис. 2 показан способ записи и управления процедурой определения времени релаксации (копия экрана монитора). При запуске программы сигналы ЯМР (реальные и мнимые компоненты) периодически отображаются в левой части экрана в шкале времени при циклическом увеличении интервала между импульсами возбуждения. Предварительный выбор оптимальной длительности импульсов возбуждения обеспечивал максимальное отношение сигнал/шум эхо-сигналов. Первый $90^{\circ}$-ный импульс обеспечивает поворот вектора намагниченности перпендикулярно направлению магнитного поля, а последующий $180^{\circ}$-ный импульс (посланный после циклически изменяемого временного интервала $t$ ) обеспечивает формирование ЯМР эхо-сигнала. Справа на рис. 2 представлены ЯМР эхо-сигналы после преобразования Фурье.

Для контроля возможностей прибора в режиме релаксометра был использован калибровочный образец, заполненный раствором воды с добавлением препарата „Магневист“, содержащий парамагнитные атомы гадолиния. Объем и концентрация парамагнитных атомов калибровочного образца соответствовали типичным параметрам исследованных далее живых тканей. 


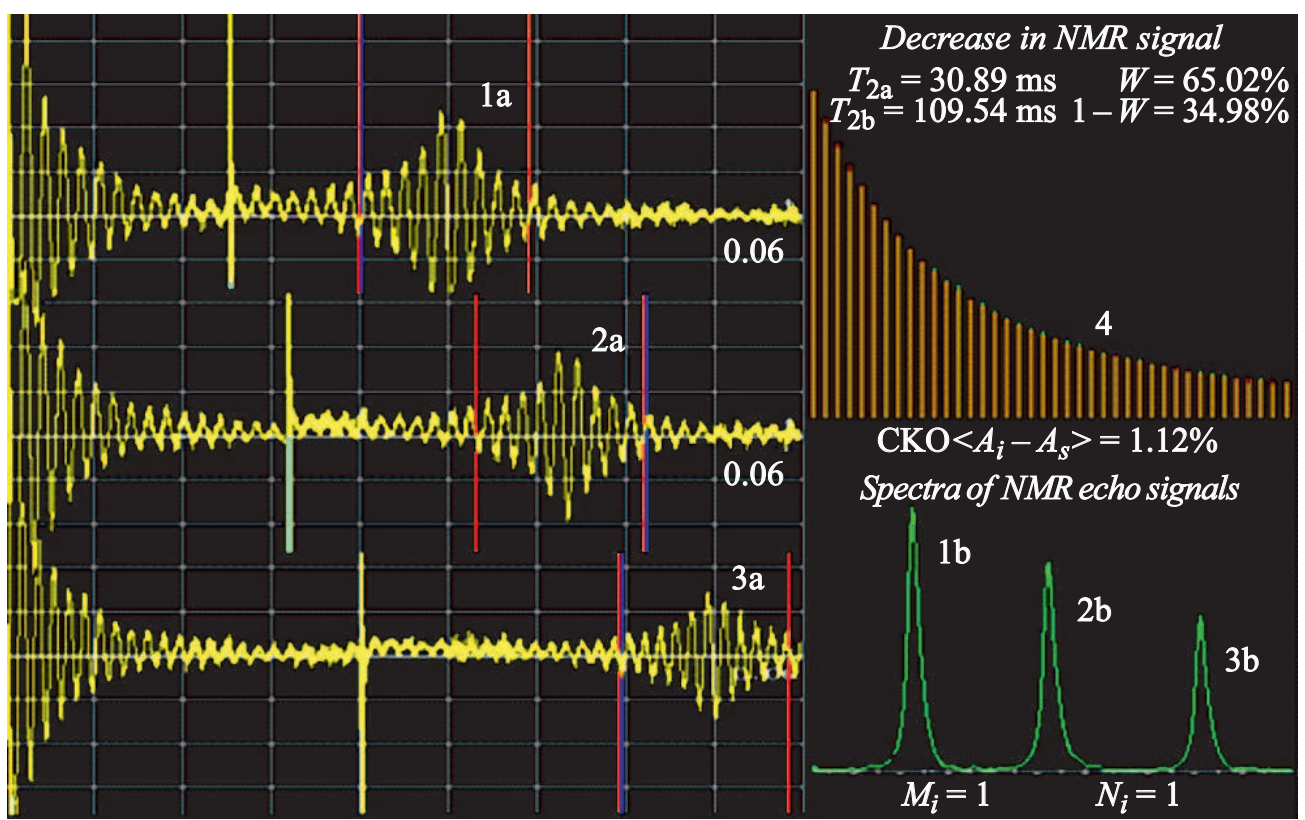

Рис. 2. Способ контроля процедуры регистрации протонной релаксации: 1a, 2a, 3a - сигналы эха при увеличении интервала времени между возбуждающими импульсами; $1 \mathrm{~b}, 2 \mathrm{~b}, 3 \mathrm{~b}$ - сигналы после преобразования Фурье; 4 - представление амплитуд сорока сигналов в виде вертикальных линий.

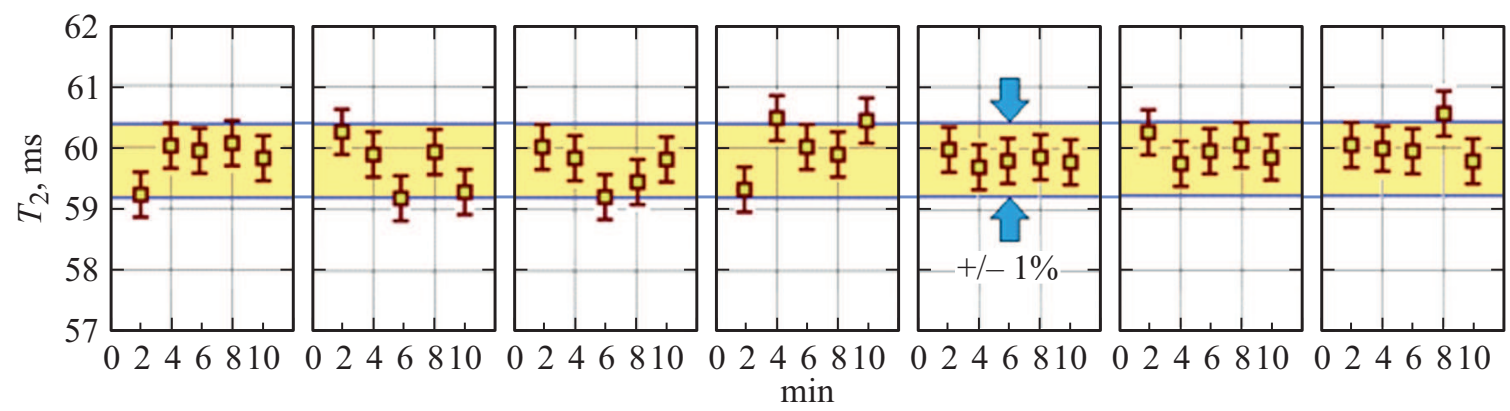

Рис. 3. Оценка стабильности работы прибора и погрешности регистрации $T_{2} \approx 59.85 \mathrm{~ms}$, выполненная для цилиндрической ампулы, заполненной раствором воды, в которую добавляли препарат „Магневист“, содержащий парамагнитные атомы гадолиния.

Было проверено, что двухимпульсный метод обеспечивает повторяемые и вполне стабильные результаты с применением метода 90-t-180, в котором программное обеспечение увеличивает временной интервал $t$ на $2 \mathrm{~ms}$ между $90^{\circ}$-ным и $180^{\circ}$-ным импульсами от $T_{E}=16 \mathrm{~ms}$ до $T_{E}=172 \mathrm{~ms}$. Цикл набора данных для вычисления $T_{2}$ содержал 40 ЯМР эхо-сигналов, для каждого из которых фурье-преобразованием формировался спектр. Амплитуды спектральных сигналов запоминались и пересылались в блок арифметики для последующего анализа.

После окончания одного цикла регистрации 40 сигналов их амплитуды выводятся на экран монитора в виде вертикальных линий совместно с расчетными данными для оценки времени релаксации. Расчетные амплитуды вычислялись методом минимизации квадратичных отклонений (как и в [11]). Сопоставление экспериментальных и расчетных амплитуд позволяет оператору кон- тролировать накопление данных и оценивать возможные изменения уровня внешних электромагнитных помех.

На рис. 3 представлены результаты тестирования прибора. Погрешность для единичного измерения контрольного образца, как правило, составляла величину $\sim 1.0 \%$, что определялась из расхождения экспериментальных и расчетных данных для 40 амплитуд ЯМР эхо-сигналов по величине среднеквадратичного разброса. Из 35 результатов (рис. 3) следует, что типичный разброс для $T_{2}$ при последовательно регистрируемых данных так же находится на уровне $\sim 1 \%$, при этом температура образца удерживалась в пределах $t=20 \pm 0.5^{\circ} \mathrm{C}$.

Уменьшение амплитуд сигналов для контрольной ампулы описывается одной экспонентой $A(t)=A_{o}$ $\times \exp \left(-t / T_{2 o}\right)$. Но в живых тканях падение амплитуды ЯМР эхо-сигналов от времени имеет более сложную 

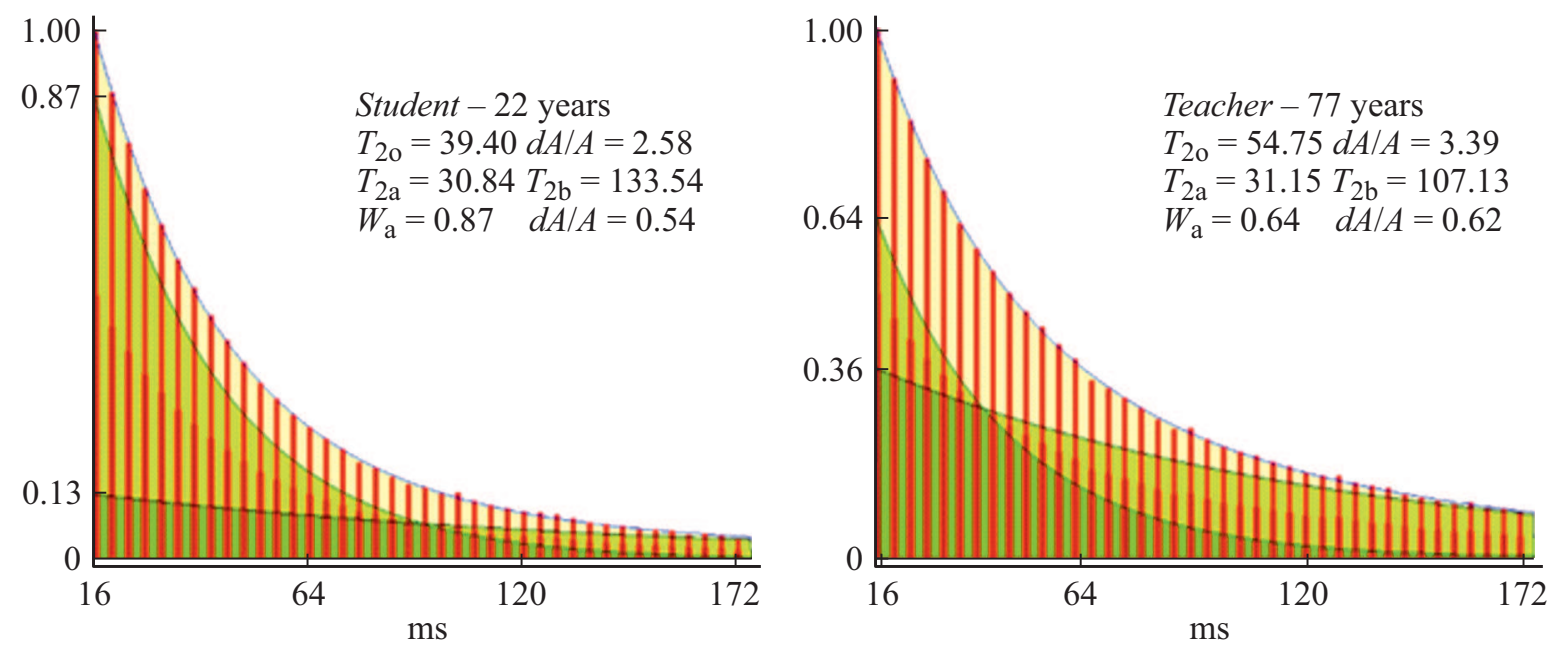

Рис. 4. Сопоставление данных об уменьшении амплитуд сигналов спинового эха для двух участников эксперимента разного возраста.

Результаты оценки времен спин-спиновой релаксации протонов воды для мышечной ткани группы студентов и преподавателей

\begin{tabular}{c|c|c|c|c|c|c|c}
\hline \multirow{2}{*}{ Возраст } & \multicolumn{2}{|c|}{$A(t)=\exp \left(-t / T_{2 o}\right)$} & \multicolumn{5}{|c}{$A(t)=W_{a} \exp \left(-t / T_{2 a}\right)+W_{b} \exp \left(-t / T_{2 b}\right)$} \\
\cline { 2 - 8 } & $T_{2 o}, \mathrm{~ms}$ & $d A / A, \%$ & $T_{2 a}, \mathrm{~ms}$ & $W_{a}, \%$ & $T_{2 b}, \mathrm{~ms}$ & $W_{b}, \%$ & $d A / A, \%$ \\
\hline $23 \pm 5$ & $40 \pm 1$ & $2.6 \pm 0.2$ & $31 \pm 1$ & $86 \pm 2$ & $120 \pm 4$ & $14 \pm 2$ & $0.51 \pm 0.05$ \\
$75 \pm 5$ & $55 \pm 2$ & $3.4 \pm 0.3$ & $32 \pm 2$ & $65 \pm 2$ & $115 \pm 4$ & $35 \pm 2$ & $0.82 \pm 0.08$
\end{tabular}

зависимость. Если для описания уменьшения амплитуд 40 сигналов от живых тканей использовать лишь одну экспоненту с $T_{2 o}$, то среднеквадратичные отклонения для сорока экспериментальных амплитуд $A_{i}$ от расчетных амплитуд $A\left(t_{j}\right)$ оказываются, как правило, на уровне $3-4 \%: d A / A \approx(3-4) \%$, где

$$
d A / A=100 \times\left(1 / A_{1}\right) \times \sqrt{\left\{\Sigma\left[A\left(t_{j}\right)-A_{i}\right]^{2}\right\} /(N-1.5)} .
$$

В программном обеспечении релаксометра был предусмотрен второй вариант математической обработки данных (как и в работах [12-14]) с использованием двух экспонент и двух весовых параметров:

$$
A(t)=A_{o}\left[w_{a} \times e^{\left(\frac{-t}{T_{2 a}}\right)}+w_{b} \times e^{\left(\frac{-t}{T_{2 b}}\right)}\right] .
$$

С использованием (3) среднеквадратические отклонения при исследовании живой ткани, как правило, уменьшались до $d A / A \approx(0.5-0.8) \%$ в зависимости от уровня шумового фона. При этом отклонения $d A / A \approx 0.7 \%$ обеспечивались вычислением трех параметров $T_{2 a}, T_{2 b}$ и $w_{a}$ с использованием метода минимизации суммы квадратичных отклонений $\Sigma\left[A\left(t_{j}\right)-A_{i}\right]^{2}$ и с учетом нормировки $\left(A_{o}=100 \% ; w_{a}+w_{b}=1\right)$.

\section{Оценка возрастных изменений}

В настоящей работе исследовалась мышечная ткань, в качестве который была выбрана muscular abductor pollicis brevis - (мышца ладони, отводящая большой палец), поскольку она являлась наиболее удобной для комфортного размещения ладони участника эксперимента в зоне регистрации настольного магнита. Для интервала времени между последовательными $90^{\circ}$-ыми импульсами было использовано $T R=3 \mathrm{~s}$. За этот интервал времени между импульсами возбуждения наблюдалось восстановление равновесной намагниченности, связанной со спиновой ориентацией протонов воды живых тканей.

На рис. 4 и в таблице представлены данные, полученные от группы студентов и преподавателей, для которых был характерен достаточно типичный образ жизни. Данные для участников эксперимента разных возрастных групп указывают на наличие возрастных изменений.

Заметим, что если данные для $T_{2 a}, T_{2 b}$ изменялись незначительно, то весовые параметры изменялись существенно. Результаты регистрировались в середине рабочего дня, чтобы минимизировать возможный дополнительный разброс данных, связанный с влиянием суточных изменений.

\section{Обсуждение результатов}

Область исследования в настоящем эксперименте охватывает разные по морфологическому строению участки мышечной ткани, которые разделены множе- 
ством биологических мембран. Определяемые данным методом параметры $T_{2 a}$ и $T_{2 b}$ являются усредненными из-за высокой трансляционной подвижности молекул воды. Согласно широко известному соотношению $\left\langle r^{2}\right\rangle=6 D t$ (уравнение Эйнштейна-Смолуховского для трехмерного перемещения), молекулы воды перемещаются на десятки микрон за интервал времени от первого импульса возбуждения до формирования сигналов спинового эха, что соизмеримо с размером отдельных клеток мышечной ткани.

В первом приближении более короткое время $T_{2 a} \approx 31(2)$ ms следует отнести к протонам воды мышечных волокон и связанных с ними клеточных структур, участвующих в обеспечении двигательных функций. Более длительное время $T_{2 b} \approx 117(5) \mathrm{ms}$ характеризует те участки ткани, в которых активных парамагнитных центров в 3-4 раза меньше. И, вероятно, эти участки ткани следует (в основном) относить к протонам воды, входящей в состав более рыхлой соединительной ткани.

Как видим (см. таблицу), параметры $w_{b}$ увеличиваются с возрастом от $15(2) \%$ до $35(2) \%$, что может указывать на разрастание объема соединительных тканей и соответственно на уменьшение весовой доли мышечных волокон. Можно предполагать, что хорошо известную возрастную потерю мышечных тканей (саркопению, [15-22]) можно численно оценивать данным методом.

Описанная методика является комфортный для практического использования и при улучшении ряда технических параметров подобных приборов данный способ может оказаться востребованным как для оценок биологического старения живых тканей, так и для оценки других патологических отклонений.

\section{Список литературы}

[1] Abragam A. The Principles of Nuclear Magnetism. Oxford at the Clarendon Press, 1961.

[2] Chizhik V.I. et al. Magnetic Resonance and Its Applications. Springer, 2014.

[3] Неронов Ю.И. Магнитный резонанс в томографии и в спектральных исследованиях тканей живого организма. Изд-во Санкт-Петербургского гос. ИТМО, 2007. 124 с.

[4] Иванов В.К., Неронов Ю.И., Иванов В.А. // Научнотехнический вестник Санкт-Петербургского гос. ИТМО. 2001. № 3. C. 201-205.

[5] Неронов Ю.И., Иванов В.К. // Научное приборостроение. 2006. T. 16. № 2. С. 105-112.

[6] Неронов Ю.И. // Известия вуз. Приборостроение. 2008. T. 51. № 10. C. 47-51.

[7] Пат. РФ № 2063702. Метод магнитно-резонансного изображения и устройство для его наблюдения / Л.А. Тютин, Ю.И. Неронов. 1996.

[8] Неронов Ю.И., Серегин А.Н. // Измерительная техника. 2011. № 1. С. 69-72. [Neronov Y.I., Seregin A.N. // Measurement Techniq. 2011. Vol. 54. N 1. P. 103-107.]

[9] Neronov Y.I., Seregin A.N. // Measurement Techniq. 2010. Vol. 53. N 8. P. 926-935.
[10] Neronov Yu.I., Aleksandrov V.S. // JETP Lett. 2011. Vol. 94. N 6. P. 418-421.

[11] Neronov Yu.I., Seregin N.N. // Metrologia. 2014. Vol. 51. N 1. P. 54-60.

[12] Kosenkov D. D., Neronov Y. I., Zolotov A. N., Seregin N.N. NMR Relaxometer for the Estimation of the Spin-Spin Proton Relaxation Time of the Living Tissue. Magnetic Resonance and its Applications. Spinus - 2018, Abstracts book. Saint Petersburg State University, Department of Nuclear Physics Research Methods. 2018. P. 77-79.

[13] Zolotov A.N., Neronov Y.I., Kosenkov D.D. An Estimate of the Change in the Spin-Spin Relaxation Time of Protons of Living Tissue Upon its Cooling. Magnetic Resonance and its Applications. Spinus - 2018, Abstracts book. Saint Petersburg State University, Department of Nuclear Physics Research Methods. 2018. P. 130-132.

[14] Неронов Ю.И., Золотов А.Н., Косенков Д.Д., Серегин Н.Н. Использование ЯМР-релаксометра для оценки изменений времени спин-спиновой релаксации протонов живой ткани в зависимости от ряда внешних условий. В сб.: Open science 2.0: Collection of Scientific Articles. Vol. 4. P. 118 123. Open Science Publishing, Lulu Press, Inc., 3101, Hillsborough St., Raleigh, North Carolina 27607, USA, 2018.

[15] Поворознюк В.В., Григорьева Н.В. Менопауза и костномышечная система. Киев, 2004. 512 с. [Povoroznyuk V.V., Grigorieva N.V. Menopause and Musculoskeletal System. K. 2004. 512 p.]

[16] Ундрицов В.М., Ундрицов И.М., Серова Л.Д. Возрастные изменения мышечной системы / В кн. Руководство по геронтологии / Под ред. акад. В.Н. Шабалина. М.: Изд-во Цитадель Трейд, 2005. С. 486-499.

[17] Ундрицов В.М., Ундрицов И.М., Серова Л.Д. // Физкультура в профилактике, лечении и реабилитации. / Под ред. С.В. Хрущева. 2009. № 4 (31). С. 7-16.

[18] Lang T., Streeper T., Cawthon P. et al.// Osteoporos Int. 2010. Vol. 21. P. 543-559.

[19] Burton L.A., Sumukadas D. // Clinical Interventions in Aging. 2010. Vol. 5. P. 217-228.

[20] Cruz-Jentoft A.J., Baeyens J.P., Bauer J.M. et al. // Age and Ageing. 2010. Vol. 39. P. 412-423.

[21] Chumlea Wm.C., Cesari M., Evans W.J. et al. // J. Nutr. Health Aging. 2011. Vol. 15. N 6. P. 450-455.

[22] Artur S.T., Colley I.D. // Intern. J. Biolog. Sci. 2012. Vol. 8. N 5. P. 731-760. 